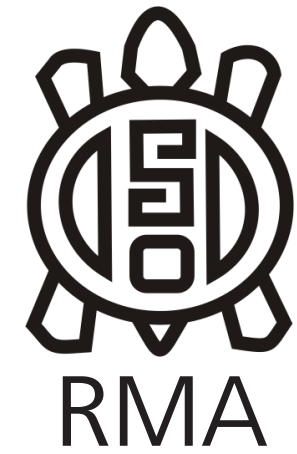

\section{Análisis formal del mortero múltiple ubicado en Cerro de los Viejos (Departamento Caleu Caleu), La Pampa, Argentina}

\author{
Formal multiple mortar analysis located in Hill of the Viejos (Caleu \\ Caleu department), La Pampa, Argentina
}

\author{
Florencia N. Paez*, Mónica A. Berón**, Eliana N. Lucero*** y \\ Manuel P. Carrera Aizpitarte****
}

*IDECU-CONICET, Museo Etnográfico "Juan Bautista Ambrosetti", Facultad de Filosofía y Letras, Universidad de Buenos Aires, Argentina. E-mail: fnatapaez@gmail.com

**IDECU-CONICET. Museo Etnográfico "Juan Bautista Ambrosetti", Facultad de Filosofía y Letras, Universidad de Buenos Aires, Argentina. Facultad de Ciencias Sociales, Universidad Nacional de Centro de la Provincia de Buenos Aires, Argentina. E-mail: monberon@retina.ar *** Museo Etnográfico "Juan Bautista Ambrosetti", Facultad de Filosofía y Letras, Universidad de Buenos Aires, Argentina. E-mail: eli.lucero2008@gmail.com ****IDECU-CONICET. Museo Etnográfico "Juan Bautista Ambrosetti", Facultad de Filosofía y Letras, Universidad de Buenos Aires, Argentina. Facultad de Ciencias Sociales, Universidad Nacional de Centro de la Provincia de Buenos Aires, Argentina. E-mail: mcarreraaizpitarte@gmail.com

\begin{abstract}
Resumen
Las actividades de molienda de vegetales y minerales se encuentran ampliamente documentadas en la provincia de La Pampa. La tecnología asociada ha sido hasta el momento de carácter transportable. En este trabajo se presenta el análisis del primer conjunto de artefactos fijos de molienda detectado en la provincia.

La presencia de tecnología fija de molienda permite abordar diversos tópicos vinculados con las sociedades del pasado, más allá de la función que específicamente cumplieron. La alta inversión de trabajo necesaria para generar los morteros y molinos fijos, sumado a la extensa vida útil que presentan, indican una preocupación por modificar y acondicionar un espacio, anticipando necesidades futuras en un lugar al que se prevé volver sucesivamente. El conjunto detectado en Cerro de los Viejos está formado por 11 morteros dispuestos en forma central, dos molinos y rasgos asociados a ellos. En su entorno se registraron diversas manifestaciones culturales, con diferentes temporalidades que abarcan desde el Holoceno Medio (ca. 6000 años) hasta tiempos históricos. Su presencia pudo alentar el continuo uso de este lugar a lo largo del tiempo promoviendo sentimientos de apropiación del espacio por los grupos que los generaron y sus descendientes. En este trabajo se consideran los aspectos morfológicos, tipológicos y sus posibles modos de uso en relación con las sociedades cazadoras- recolectoras del semidesierto pampeano.
\end{abstract}

Palabras clave: Mortero múltiple; Cazador-recolector; Funcionalidad; Transgeneracional; La Pampa.

\begin{abstract}
The grinding activities of vegetables and minerals are widely documented in La Pampa province. The associated technology is transportable in nature. In this work the analysis of the first set of fixed multiple grinding artifacts detected in the province is presented.

The presence of this type of materiality let us approach various topics related to the past societies, beyond the function that they specifically fulfilled. The high investment of work necessary to generate the fixed mortars and grinders, added to its long useful life, indicate an anticipated concern to modify and condition for future needs a place to which is expected to return successively.

The set detected in Cerro de los Viejos comprises 11 mortars arranged in a central way, 2 grinder and some features associated with them. Various cultural manifestations were recorded in its surroundings, with different temporalities ranging from the Middle Holocene (ca. 6000 years) to historical times. Their presence could encourage the continued use of this place over time, by promoting feelings of appropriation of space by the groups that generated them and their descendants. In this work the morphological, typological aspects and their possible modes of use are considered in relation to the hunter-gatherer societies of the Pampean semi-desert.
\end{abstract}

Key words: Multiple mortars; Hunter-gatherers; Functionality; Transgenerational; La Pampa. 
La evidencia arqueológica recuperada en la provincia de La Pampa, Argentina, permite comprender la trayectoria de los grupos de cazadores-recolectores en un entorno semidesértico durante el Holoceno. Los primeros asentamientos en la región ofrecen registros efímeros para el Holoceno Temprano y Medio, en 4 loci principales: Casa de Piedra 1, Tapera Moreira 1, El Carmel y Cerro de los Viejos (ca. 8620-4590 años AP). Los grupos se asentaron en espacios donde los recursos estaban concentrados, pero circularon en paisajes con topografías variadas. En el Holoceno Tardío se estabilizan las condiciones climáticas actuales, se registra un crecimiento demográfico y se intensifica la explotación de los recursos locales. Aparecen estructuras formales de entierro y se incrementa la presencia de elementos extraregionales como un signo de interacciones a gran escala. En este contexto, progresivamente se incorporan nuevas tecnologías como alfarería, molienda, manufactura de adornos malacológicos, entre otras.

Las estructuras de molienda presentes en contextos de cazadores-recolectores, han sido tradicionalmente interpretadas como evidencia directa o indirecta de la recolección y preparación de recursos vegetales (Berón, 2004; Jackson, 2004; Tapia y Charlin, 2004; Vecchi y González, 2018; entre otros). Sin embargo, a partir de datos arqueológicos (Babot y Apella, 2001; Babot, 2004; Giovannetti, 2009) y etnográficos (Adams, 1999), se reconocen otras funciones como, por ejemplo, la preparación de pigmentos (vegetales, animales y minerales) y alimentos de origen animal (Babot, 1999; Schlagner, 1991). Sumado a lo anterior, estas estructuras conforman conjuntos artefactuales altamente significativos para comprender los contextos arqueológicos asociados, desde diversos puntos de vista, tecnológico, socioeconómico, demográfico e ideológico (Giovannetti, 2009; Jackson, 2004). En este sentido, se trata de una materialidad que posee un carácter transversal para el conocimiento de las sociedades del pasado con una característica que los identifica: su transgeneracionalidad, o en palabras de Pastor (2015), con potencial multi-generacional. Al tratarse de artefactos con larga vida útil, con la posibilidad de una reutilización alternando etapas de uso y desuso de las oquedades (Pino, Troncoso, Belmar y Pascual, 2018).

Si se considera que la cultura material es un transmisor de información sobre los comportamientos de los individuos que la generaron, al abordar su estudio comprendemos que ésta, no solo incluye a los objetos per se, sino también a las prácticas cotidianas, espacios y relaciones sociales, de los cuales tales objetos formaban parte. También incluye los diferentes significados que lo material adquiere en la mutua interacción hombre - objeto a lo largo del tiempo (Gosden y Marshall, 1999; Moreyra, 2011). Entonces, es válido sostener que, si las prácticas sociales, relaciones y espacios mutan y se resignifican, los objetos también lo harán. Así, las estructuras de molienda, como componentes de la materialidad, interactúan y participan activamente en las trayectorias de vida de las personas (Keane, 2005; Miller, 2005; Tilley, 1999).

Se ha abordado el estudio de las estructuras de molienda emplazadas en Cerro de los Viejos bajo esta premisa y considerando además cómo ellas se insertan en un paisaje conformado por diversas manifestaciones culturales, con diferentes temporalidades. La singularidad de los morteros de Cerro de los Viejos, radica en su excepcionalidad para la provincia de La Pampa y posiblemente para la región pampeana. Los objetivos de este trabajo son ampliar el conocimiento sobre las estructuras fijas de molienda, ubicadas en la localidad arqueológica de Cerro de los Viejos, realizar una caracterización tipológica y morfológica y evaluar sus posibles modos de uso alternativos, en un paisaje que actuó como un ecorefugio de carácter referencial a lo largo de varios milenios.

\section{Características del área de estudio}

Cerro de Los Viejos (S38 $28^{\prime} 33,3^{\prime \prime} ;$ O64 $\left.26^{\prime} 21,9^{\prime \prime}\right)$ se ubica en el departamento de Caleu Caleu, en el sector sureste de la provincia de La Pampa, a ca. $25 \mathrm{~km}$ de la localidad de Cuchillo-Có y ca. $65 \mathrm{~km}$ de la localidad de La Adela (Figura 1). El cerro es un afloramiento de forma elíptica de 1,5 km por $1 \mathrm{~km}$, conectado con depósitos sedimentarios recientes e integra el basamento ígneometamórfico de la provincia de La Pampa (Tickyj, Dimieri, Llambias y Sato, 1997). Es un macizo granítico que alcanza los 216 msnm, siendo la elevación de mayor altura en la zona. Esta topografía se destaca en el paisaje y le confiere características particulares al ambiente de esta área.

La localidad arqueológica de Cerro de los Viejos se ubica en el área de estudio denominada Bajos sin Salida. La característica genética de esta unidad está dada por la acción de los procesos vinculados a los paleocauces del río Colorado, cuyos escurrimientos hídricos concentrados, dieron lugar a un relieve posteriormente sometido al modelado eólico. Es una de las pocas zonas del mundo cuyas cotas de altura están por debajo del nivel del mar. Estudios paleoambientales realizados en la Salina

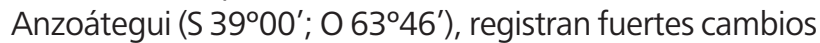
en los niveles de agua y en las condiciones ambientales entre fines del Holoceno Medio y comienzos del Tardío (Schäbitz, 1994). El mayor interés de estos ambientes es que son dinámicos y contienen cuerpos de agua, cuyo régimen puede ser transitorio o permanente. Esto último genera un ecosistema particular, con vegetación y fauna diferentes del entorno, lo cual posiblemente resultó en un atractivo para las poblaciones del pasado (Carrera Aizpitarte, Luna, Aranda y Berón, 2013).

El área se caracteriza por presentar un índice hídrico correspondiente a un clima semiárido (INTA, 1980),

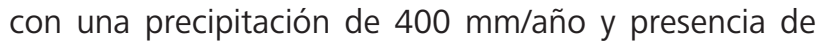
suelos arenosos y pedregosos. Fitogeográficamente se 
encuentra ubicado dentro de la Provincia del Espinal, cuya principal característica es la presencia de bosque xerófilo bajo, denso o abierto con arbustos perennes y pastizales (Cabrera, 1971).

\section{Antecedentes}

En 1973 Ernesto Piana realizó una primera prospección en Cerro de los Viejos, donde relevó seis estructuras de piedra que interpretó como represas (Figura 2). Seis años más tarde, realizó una campaña arqueológica en el lugar cuyos objetivos se centraron en el análisis de las técnicas constructivas de las represas y en el registro de evidencias arqueológicas: artefactos líticos, fragmentos cerámicos, restos óseos quemados, morteros móviles, la mayor parte hallados en superficie (Piana, 1981). En esta oportunidad se relevaron también los morteros múltiples. En cuanto a las estructuras de piedra, la propuesta interpretativa consistía en su vinculación con los arreos de ganado vacuno desde la provincia de Buenos Aires hacia Chile, durante el denominado "ciclo del ganado", entre los siglos XVII y XIX (Mandrini, 1984, 1991; Palermo, 1986, 1991; Pedrotta, 2016). Las represas constituían un modo artificial de crear reservorios de agua para abrevar animales en la época en que los aborígenes realizaban arreos de ganado hacia Chile y empleaban este lugar como posta y/o descanso para reponer fuerzas. El Cerro de los Viejos estaba ubicado en una de las principales rastrilladas o caminos indígenas. Esta idea está apoyada por abundantes datos etnohistóricos (Mandrini 1984) y arqueológicos (Goñi, 1986-87; Madrid, 1991, Pedrotta 2016), que relatan la importancia de estas construcciones en las travesías entre el $\mathrm{O}$. de la provincia de Buenos Aires y los pasos cordilleranos, cuando se comerciaba con Chile ganado vacuno y caballar, ya fuera cimarrón o tomado de las estancias.

Entre los años 2004 y 2009 dos de los autores (MB y MCA) realizaron incursiones esporádicas a la Localidad Cerro de los Viejos (CLV) y como resultado se registró un enterratorio (CLV1), cuyo cráneo fue recuperado de manos de un huaquero y datado. Se obtuvo un fechado de 3035+/-25 años AP (UGAMS 4417). A partir del año 2017, en el marco de un proyecto ASETUR (línea de financiamiento nacional destinada al apoyo del Sector Turismo) dirigido por la Dirección de Patrimonio de la Secretaría de Cultura de la provincia de La Pampa, comenzaron tareas de relevamiento, prospección y excavaciones sistemáticas. Hasta el momento fueron detectadas diversas manifestaciones arqueológicas, entre ellas artefactos líticos, fragmentos cerámicos, restos faunísticos en superficie, un contexto doméstico y dos enterratorios en estratigrafía (CLV 2 y 3). Ambos entierros fueron hallados sobre el perfil de un cañadón de erosión aluvional que contornea el cerro. El entierro CLV 2 se encontraba cubierto por siete piedras lajas, y es de tipo primario simple. Se trata de un individuo adulto masculino colocado en posición de cubito lateral derecho, que presentaba un importante ajuar mortuorio, entre los brazos, a la altura del pecho. Por su parte, el entierro CLV 3 corresponde a un entierro primario simple. Debido a la compactación de los sedimentos y el escaso tiempo disponible se decidió extraerlo en bloque. Los análisis de ambos entierros y del contexto doméstico se encuentran en proceso, aunque los primeros datos recabados indican que CLV2 corresponde al Holoceno Medio (ca. 6000 años AP; Berón, 2019). Tanto su cronología como las características del ajuar mortuorio remiten a otro caso similar correspondiente al sitio 1 de Casa de Piedra, datado en $6080 \pm 120$ años AP (Gradín et al., 1984). Estas nuevas evidencias se suman a las estructuras fijas de molienda y las represas relevadas por Ernesto Piana y colaboradores en el año 1979.

\section{Cuestiones metodológicas y conceptuales}

Para abordar el análisis del conjunto de molienda denominado CLV4, el primer paso fue establecer la diferencia entre molinos planos y morteros a partir de su morfología. Los molinos, son artefactos de

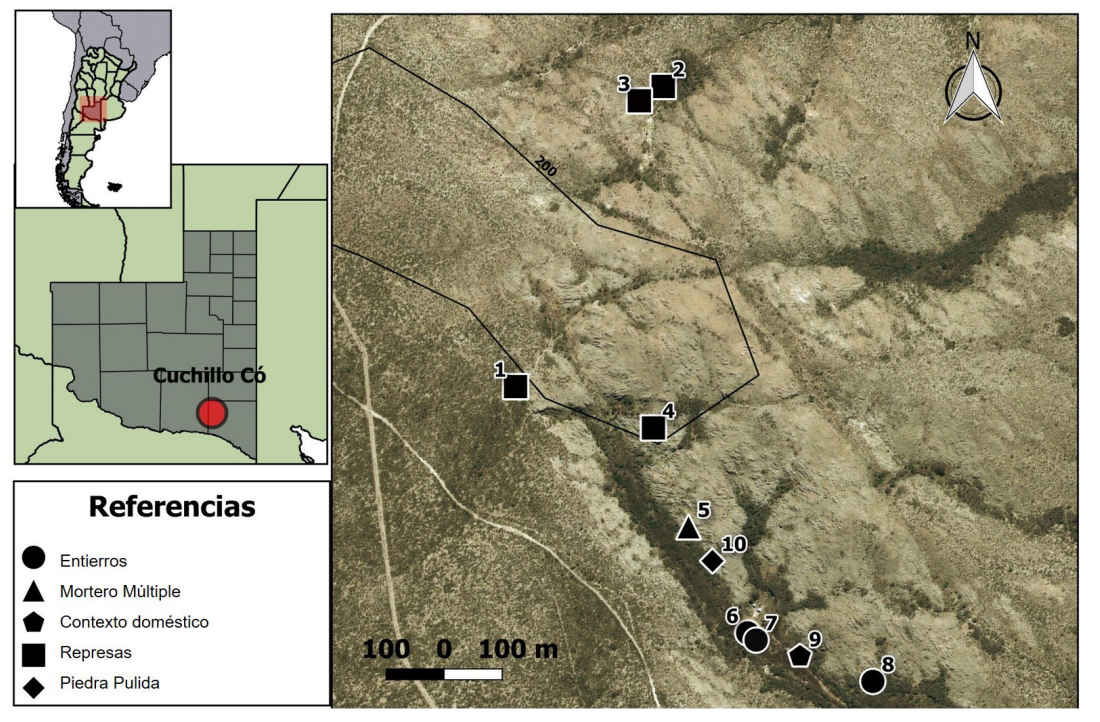

Figura 1. Localización de Cerro de los Viejos. Referencias, 1: Represa $N^{\circ} 1 ; 2$ : Represa $N^{\circ} 2$; 3: Represa $N^{\circ} 3$; 4: Represa N4; 5: CLV4 Mortero Múltiple; 6: CLV2 Entierro; 7: CLV1 Entierro; 8: CLV3 Entierro; 9: CLV5: Contexto doméstico; 10: Piedra pulida "El Tobogán".

Figure 1. Location of Cerro de los Viejos. References, 1: Dam N¹; 2: Dam N²; 3: Dam N³; 4: Dam N4; 5: CLV4 Multiple Mortar; 6: CLV2 Burial; 7: CLV1 Burial; 8: CLV3 Burial; 9: Domestic context; 10: Polished stone "El Tobogán". 
molienda pasivos, de forma generalmente elongada y se caracterizan por presentar una zona activa dispuesta en sentido horizontal, formada por una depresión cóncava o superficie plana (Babot, 2007; Giovannetti, 2009). Los morteros, también artefactos de molienda pasivos, se diferencian de los molinos porque su zona activa está conformada por una cavidad con predominio vertical, suele ser profunda y de boca circular o sub-circular (Babot, 2007; Giovannetti, 2009; Nardi y Chertudi, 1969). Siguiendo a Giovannetti (2009) utilizamos la denominación de mortero múltiple para hacer referencia al conjunto de morteros y molinos, manufacturados sobre un gran bloque de roca inamovible, caracterizado por presentar las oquedades en superficies rocosas planas, horizontales o semi horizontales.

Las variables que han sido consideradas para el análisis y descripción del conjunto de molienda, han sido tomadas y adaptadas de Babot $(2004,2007)$ y Giovannetti (2009). Las distintas categorías son:

Roca soporte: alude a la naturaleza geológica de la roca que conforma el área de molienda.

Movilidad: posibilidad de transportar la roca que contiene la unidad/es de molienda.

Unidad de análisis: hace referencia a cada uno de los elementos del conjunto de molienda (mortero o molino plano), registrados con números consecutivos y analizados de manera individual.

Tipo de unidad: indica si la unidad es de carácter aislada (una) o compuesta (dos o más), cuando diferentes unidades se combinan entre sí.

Combinación: se refiere a la cantidad de elementos dentro de un conjunto de molienda (dobles, triples, cuádruples, etc.).

Agrupamiento: Cuando se trata de unidades combinadas, se indica, según la numeración otorgada, cuáles son las unidades agrupadas entre sí.

Rasgos asociados: se refiere a la presencia de canaletas, superficies de apoyo, rebajes, pulimentos, entre otros, relacionados con las unidades de análisis.

Otras variables que se consideraron durante el relevamiento de cada unidad fueron: en los morteros, diámetro mayor de la boca, diámetro de la base y profundidad; en molinos, su ancho y largo máximo. También se registraron macroscópicamente, rasgos indicativos para establecer la posible posición y número mínimo de operarios simultáneos (dimensiones, sección de las oquedades, pendientes, pulidos exteriores; según Babot, 2007 y Pastor, 2015). Los dos diámetros (boca y base) del mortero, permiten conocer la morfología de la oquedad en planta, lo que, sumado a la profundidad, permiten esquematizar su perfil. Debido a las significativas variaciones de tamaño entre las unidades de molienda, se siguió la propuesta de Pastor (2015) para grupos tipológicos. El autor incluye "dentro del Grupo tipológico 1 (GT1), a los morteros profundos, útiles de boca subcircular, sección parcialmente cónica y una profundidad generalmente superior a los $10 \mathrm{~cm}$. El GT2 (morteros playos), también poseen bocas subcirculares pero su profundidad no suele superar los $10 \mathrm{~cm}$ aunque el límite de separación entre ambos grupos en torno a los $10 \mathrm{~cm}$ de profundidad no es absoluto y finalmente, el GT3 incluye a los molinos o conanas" (Pastor, 2015:308).

Estos útiles pasivos también presentan variaciones en sus formas y tamaños y eventualmente se encuentran asociados a las oquedades en los morteros múltiples. Además, el conjunto fue georreferenciado, fotografiado y esquematizado mediante mapeo centimétrico. Este registro posibilitó apreciar al conjunto en escala, su ordenamiento y las relaciones espaciales entre cada unidad de molienda dentro del soporte rocoso.

Con el objetivo de evaluar la posible funcionalidad del conjunto se tomaron muestras del agua de lluvia contenida en los morteros con el fin de observar la presencia de microrrestos vegetales. El muestreo fue realizado por la Dra. G. Musaubach en el año 2009 y el análisis arqueobotánico se efectuó en el año 2017. Según el protocolo descripto en Musaubach (2014), las muestras fueron montadas en portaobjetos y luego observadas bajo microscopio de luz polarizada y objetivo de 40X de la serie JPL-1350. El registro microfotográfico se realizó con una cámara digital Nikon Coolplix L18. Las identificaciones taxonómicas se llevaron a cabo mediante la utilización de claves de identificación basadas parcialmente en el International Code for Phytolith Nomenclature 1.0 (Madella, Alexandre y Ball, 2005), el International Code for Starch Nomenclature (2011) y en material de referencia publicado por Bertoldi de Pomar (1975), entre otros. No se realizó raspado directo de las oquedades por encontrarse en ese momento llenos de agua y porque algunos protocolos de trabajo tampoco recomiendan dicha toma de muestras en morteros expuestos permanentemente a agentes tafonómicos (Petrucci, 2016). Además, el proceso de investigación arqueobotánico se discontinuó, aunque se planea retomarlo próximamente y realizar otro tipo de muestreos recomendados en Giovannetti (2013).

\section{Descripción del Conjunto de Molienda}

El conjunto de molienda se ubicada en el sector suroeste del Cerro de los Viejos, sobre una de las laderas principales de acceso, desde donde se posibilita una buena observación del entorno (Figura 2). Este sector no se encuentra delimitado por construcciones o muros naturales y es uno de los puntos de conexión entre la zona de ascenso al cerro y el piedemonte que concentra los recursos (Figura 2). A escasos metros del mortero múltiple hay una roca saliente con marcada inclinación 
y acentuado pulimento, conocida localmente como "el tobogán" (Figura 2).

El mortero múltiple se compone de 11 oquedades y 2 molinos planos, distribuidos en una superficie de ca. 1,50 $\mathrm{m}$ de ancho por $3 \mathrm{~m}$ de largo (Figura 3). El soporte rocoso, es el basamento granítico propio de la formación del cerro. Las oquedades son circulares con vista en planta y cupuliformes en perfil, dado que sus paredes tienden a ser cilíndricas y presentan un diámetro de boca bastante similar al de la base. Existe una amplia diversidad entre los diámetros de las bocas, las bases y las profundidades de cada mortero, alcanzando los más grandes $31,5 \mathrm{~cm}$ de diámetro por $37,9 \mathrm{~cm}$ de profundidad y los más pequeños $7 \mathrm{~cm}$ de diámetro por 1,5 cm de profundidad (Tabla 1). Los molinos son elongados, presentan superficies pulidas, cavidades planas a semi-cóncavas y también se diferencian entre sí (Tabla 1). Dada estas características, las unidades se han agrupado siguiendo la propuesta de grupos tipológicos realizada por Pastor (2015). Fueron identificados 6 morteros correspondientes al GT1 ( ${ }^{\circ} 1$; $2 ; 5 ; 8 ; 9$ y 10), 5 al GT2 (N³; 4; 6; 7 y 11) y 2 molinos en el GT3 (Figura 4). Por otro lado, se puede observar que algunas oquedades han sido manufacturadas contiguas, a tal punto que alguno de sus laterales ha desaparecido (Figura 3). En estos casos, donde morteros individuales han conformado uno compuesto, indicamos si se trata de una combinación de dos o más unidades y como se da el agrupamiento entre ellos (Tabla 2). Como rasgos asociados, se señala la presencia de dos canaletas. El mortero $N^{\circ} 10$, que tiene la particularidad de ser el más grande, presenta una canaleta, que nace desde la superficie de la roca soporte y se extiende de manera inclinada hasta conectarse con la boca del mortero (Figura 3). La otra canaleta es interna, y conecta el Mortero $N^{\circ} 8$ con el mortero compuesto por 3 unidades agrupadas $\left(N^{\circ} 1, N^{\circ} 2\right.$ y $\left.N^{\circ} 3\right)$, provocando el trasvasamiento de líquido del primero al segundo cuando el nivel lo permite.

Los bordes de la boca de cada mortero se encuentran pulidos y se puede observar en su interior inclinaciones en algunos de sus laterales. Los molinos de este conjunto se caracterizan por presentar leves depresiones, manifiestan pulimento en su zona activa y la superficie de la roca soporte presenta sectores que son muy suaves al tacto. Todo esto es relevante a la hora de pensar posiciones y gestos de molienda considerando que los usuarios deben sentarse o arrodillarse y no molestarse entre sí. En base a las dimensiones de la roca, la cantidad de unidades de molienda y su disposición, se ha calculado un mínimo de 3 y un máximo de 5 operadores que podrían haber estado trabajando en simultáneo (Figura 4).

El análisis de microrrestos realizado sobre las muestras de agua recolectadas en el año 2009 indica la presencia de fitolitos de gramíneas con morfologías variadas. Entre los macrofitolitos se encontraron células buliformes, bases de pelos, elongados y tricomas. Entre los fitolitos de las células cortas, los morfotipos hallados son saddle, rondel, trapezoidal y bilobados de los tipos Panicoide simple, irregular, largo y Stipa. En el caso de las células cortas se destacan los saddles, asignables a la subfamilia Chloridoide, con via fotosintética de cuatro carbonos (C4), los bilobados tipo Stipa (C3) y panicoides (C4) y rondels asignables a la subfamilia de gramíneas Festucoide (C3). Se observaron restos de microorganismos: insecto y posible diatomea. La especialista consideró que los procesos tafonómicos desvirtúan los resultados precisos, no permitiendo delimitar un uso específico para las oquedades (Musaubach, 2017).

\section{Discusión}

Los artefactos de molienda en general, y los morteros múltiples en particular, han sido protagonistas de grandes discusiones sobre su funcionalidad, ya que tradicionalmente fueron considerados como indicadores del modo de subsistencia de un grupo social (Babot 2009). La mayoría de las interpretaciones clásicas establecieron su función en relación con la molienda de recursos vegetales (De Aparicio, 1925; Bruch, 1911) mientras que otras, consideraron funciones alternativas como la de contener agua de lluvia (Vignati, 1931) o como objetos activos en ceremonias y rituales prehispánicos (Ledesma, 1961).

Estudios actuales etnoarqueológicos y funcionales sugieren en cambio, que no existe una relación simple y directa entre la forma y función de los artefactos de molienda. Estos estudios señalan que la función puede variar durante la vida útil del artefacto, implicando el procesamiento de recursos de diverso origen y tambiénque diseños completamente similares, son utilizados con fines distintos (Adams, 1999; Babot, 2004; Flannery, 1986; Hayden, 1987; Mansur-Franchomme, Orquera y Piana, 1987-88; Núñez y Santoro, 1990; Wright, 1994). Además de este potencial multifuncional (Wright, 1994), los conjuntos fijos de molienda son una materialidad caracterizada por su transgeneracionalidad, al ser artefactos con larga vida útil y de elaboración costosa. Dadas estas características, consideramos que los morteros múltiples no son artefactos inertes sobre los cuales se hacen cosas, sino que son objetos activos, que al igual que las personas y los espacios, se transforman y resignifican con el paso del tiempo.

Cerro de los Viejos es el ejemplo de cómo en un territorio los grupos sociales cambian, son reemplazados y nuevas generaciones resignifican el espacio preexistente a partir de su propia dinámica y devenir cultural (Hermosilla Osorio, 2017). Las diversas manifestaciones culturales identificadas en el cerro, tanto por Piana como por nuestro equipo de investigación, dan cuenta de que este lugar fue utilizado para distintos fines a lo largo del tiempo. Durante el Holoceno Medio y comienzos del Holoceno tardío fue elegido como lugar para la inhumación 


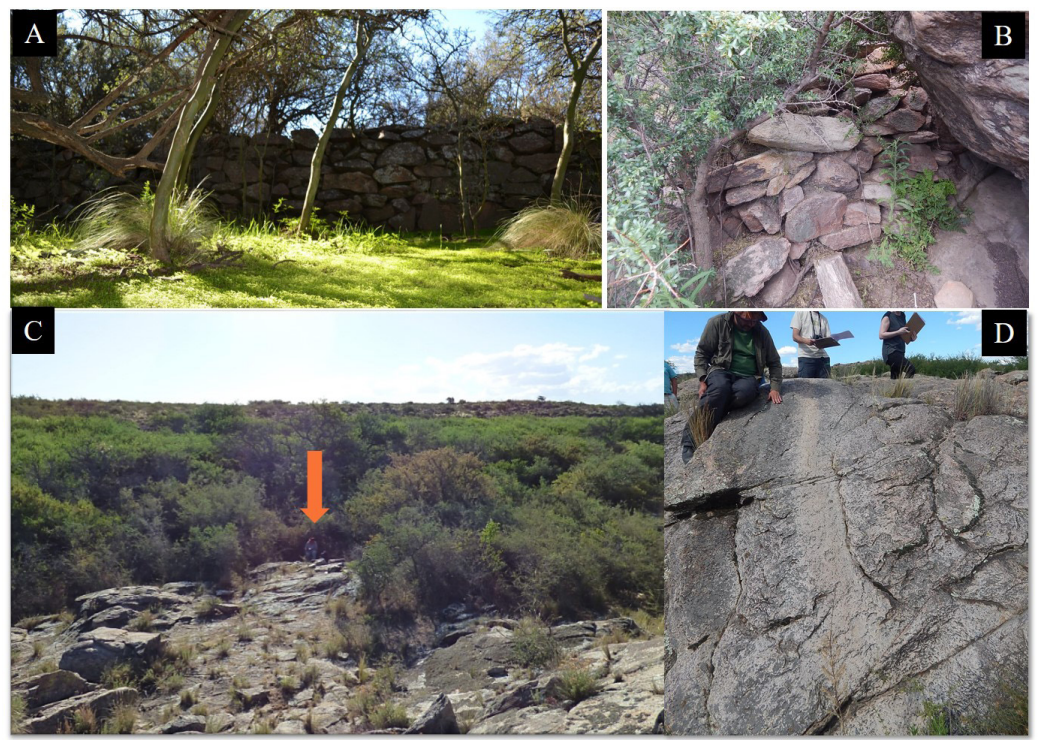

Figura 2. Referencias, $\mathrm{A}$ : Represa $\mathrm{N}^{\circ} 2$; $\mathrm{B}$ : Represa $\mathrm{N}^{\circ} 4$; C: Ubicación del mortero múltiple; D: Piedra Pulida "El Tobogán".

Figure 2. References, $A: \operatorname{Dam} N^{\circ} 2 ; B: D a m N^{\circ} 4 ;$ $C$ : Location of the multiple mortar; D: Polished Stone "El Tobogán".

de los muertos. Probablemente a esta cronología correspondan también algunos de los artefactos que fueron recuperados, tanto en forma superficial como en estratigrafía, a través de excavaciones. En tiempos históricos, con una situación político-social y económica diferente, se habría aprovechado la topografía del cerro para la generación de aguadas artificiales con las cuales facilitar el traslado y comercio de ganado desde la región pampeana hasta los ambientes cordilleranos. En el caso de las estructuras fijas de molienda es difícil estipular una antigüedad precisa debido a la carencia de elementos datables. En el entorno inmediato del mortero múltiple no hay acumulaciones sedimentarias, pero se realizaron sondeos a $100 \mathrm{~m}$ aproximadamente, sin resultados. No obstante, su similitud con estructuras identificadas en otras regiones de Argentina y Chile, permitiría asignarlas a momentos previos al contacto hispano-indígena (Babot, 2004; Giovannetti, 2009; Hermosilla y Ramírez, 1985; Jackson, 2004; Pastor, 2015; Petrucci, 2016).

Realizar una comparación a nivel regional es imposible. El mortero múltiple ubicado en Cerro de los Viejos, es hasta el momento el único detectado en la provincia de La Pampa y posiblemente sea el único con estas características en la Región Pampeana. Esto, llevó a realizar una búsqueda bibliográfica a fin de conocer y comprender cómo fue abordado el estudio de los conjuntos de molienda en otras regiones de Argentina y países limítrofes. Principalmente nos centramos en los trabajos realizados en el Noroeste Argentino (Babot, 2004, 2007, 2009; Giovannetti, 2009, 2013; Petrucci, 2016), Sierras Centrales (Pastor, 2010, 2015) y Chile (Hermosilla y Ramírez, 1985; Jackson, 2004; Planella Ortiz, Santander Horta y McRostie Bustamante, 2017). Esta recopilación bibliográfica sirvió para delinear generalidades y atender a ciertas particularidades que fueran señaladas por los autores y que se registran también en el conjunto de Cerro de los Viejos. Al igual que la mayoría de los morteros múltiples registrados en las regiones antes mencionadas, CLV4 es un conjunto fijo de molienda donde aparecen asociados morteros -cupuliformes de boca circular con amplia variabilidad en sus medidas- con molinos planos. No obstante, presenta

\begin{tabular}{|c|c|c|c|c|c|c|c|c|}
\hline \multirow{2}{*}{$\begin{array}{c}\text { CLV4 } \\
\text { Mortero } \\
\text { Múltiple } \\
\text { Unidad N }\end{array}$} & \multicolumn{3}{|c|}{ Morteros } & \multicolumn{2}{|c|}{ Molinos planos } & \multirow{2}{*}{ Perfil } & \multirow{2}{*}{$\begin{array}{l}\text { Profundidad } \\
\text { (cm) }\end{array}$} & \multirow{2}{*}{ GT } \\
\hline & Boca & $\begin{array}{c}\text { Diámetro } \\
\text { de boca } \\
\text { (cm) }\end{array}$ & $\begin{array}{c}\text { Diámetro } \\
\text { de base } \\
\text { (cm) }\end{array}$ & $\begin{array}{c}\text { Ancho } \\
\text { Máximo } \\
\text { (cm) }\end{array}$ & $\begin{array}{c}\text { Largo } \\
\text { Máximo } \\
\text { (cm) }\end{array}$ & & & \\
\hline Mortero $N^{\circ} 1$ & Circular & 28 & 26,5 & - & - & Cupuliforme & 33 & 1 \\
\hline Mortero $\mathrm{N}^{\circ} 2$ & Circular & 13,5 & 12 & - & - & Cupuliforme & 13 & 1 \\
\hline Mortero $\mathrm{N}^{\circ} 3$ & Circular & 10 & 9 & - & - & Cupuliforme & 5,5 & 2 \\
\hline Mortero $\mathrm{N}^{\circ} 4$ & Circular & 9,5 & - & - & - & - & 2,5 & 2 \\
\hline Mortero $N^{\circ} 5$ & Circular & 14 & 13,5 & - & - & Cupuliforme & 24,7 & 1 \\
\hline Mortero $\mathrm{N}^{\circ} 6$ & Circular & 7 & - & - & - & - & 1,5 & 2 \\
\hline Mortero $\mathrm{N}^{\circ} 7$ & Circular & 8 & - & - & - & - & 4 & 2 \\
\hline Mortero $\mathrm{N}^{\circ} 8$ & Circular & 12 & 10,5 & - & - & Cupuliforme & 12,8 & 1 \\
\hline Mortero $N^{\circ} 9$ & Circular & 21,1 & 19 & - & - & Cupuliforme & 30,2 & 1 \\
\hline Mortero $\mathrm{N}^{\circ} 10$ & Circular & 31,5 & 30,2 & - & - & Cupuliforme & 37,9 & 1 \\
\hline Mortero $\mathrm{N}^{\circ} 11$ & Circular & 10 & - & - & - & - & 3,5 & 2 \\
\hline Molino $\mathrm{N}^{\circ} 1$ & - & - & - & 17 & 35 & $\begin{array}{c}\text { Semi- } \\
\text { cóncavo }\end{array}$ & - & 3 \\
\hline Molino $\mathrm{N}^{\circ} 2$ & - & - & - & 14 & 22 & $\begin{array}{c}\text { Semi- } \\
\text { cóncavo }\end{array}$ & - & 3 \\
\hline
\end{tabular}

Tabla 1. Medidas y secciones de las unidades que componen el mortero múltiple de Cerro de los Viejos.

Table 1. Measurements and sections of the units that make up the multiple mortar of Cerro de los Viejos. 
Tabla 2. CLV4 Mortero múltiple.

Table 2. CLV4 Multiple mortar.

\begin{tabular}{|c|c|c|c|c|c|c|}
\hline \multirow[t]{13}{*}{$\begin{array}{c}\text { CLV4 } \\
\text { Mortero } \\
\text { Múltiple }\end{array}$} & & & & & & \\
\hline & \multicolumn{2}{|c|}{ Roca Soporte } & \multirow[b]{2}{*}{ Unidad } & \multirow{2}{*}{$\begin{array}{l}\text { Tipo de } \\
\text { Unidad }\end{array}$} & \multirow[b]{2}{*}{ Combinación } & \multirow[b]{2}{*}{ Agupamiento } \\
\hline & $\begin{array}{l}\text { Naturaleza } \\
\text { Geológica }\end{array}$ & Movilidad & & & & \\
\hline & \multirow{10}{*}{$\begin{array}{l}\text { Basamento } \\
\text { granitoide }\end{array}$} & \multirow{10}{*}{$\begin{array}{l}\text { Conjunto no } \\
\text { transportable }\end{array}$} & Mortero & Compuesto & Triple & $\begin{array}{c}\mathrm{N}^{\circ} 1, \mathrm{~N}^{\circ} 2, \mathrm{~N}^{\circ} \\
3\end{array}$ \\
\hline & & & Mortero & Compuesto & Doble & $\mathrm{N}^{\circ} 5, \mathrm{~N}^{\circ} 9$ \\
\hline & & & Mortero & Aislado & - & - \\
\hline & & & Mortero & Aislado & - & - \\
\hline & & & Mortero & Aislado & - & - \\
\hline & & & Mortero & Aislado & - & - \\
\hline & & & Mortero & Aislado & - & - \\
\hline & & & Mortero & Aislado & - & - \\
\hline & & & Molino & Aislado & - & - \\
\hline & & & Molino & Aislado & - & - \\
\hline
\end{tabular}

la característica particular que ha señalado Giovannetti (2009) para los morteros múltiples de El Shincal y Petrucci (2016) en Rincón Chico, ya que fueron registradas oquedades que, al haber sido manufacturadas contiguas, alguno de sus laterales ha desaparecido fundiéndose en una sola. Estas estructuras combinadas que agrupan dos o más unidades, son complejas y aún se desconoce su funcionalidad. Su morfología quizá se deba a una especificidad funcional, a la intensidad, frecuencia de uso, a la preparación de un molido especial que requiera un tipo de molienda en particular o bien que hayan sido utilizadas para otros fines, ya que no todas las piedras con oquedades presentan una relación necesaria con el ámbito de la molienda (Babot, 2017).

Otra característica que presenta el conjunto de CLV4, es la presencia de canaletas asociadas. Si bien en la bibliografía es mencionada la presencia de canaletas en los morteros fijos, fue poca la atención que recibió está particularidad en la arqueología argentina, quedando su función relegada al posible trasvase de líquidos vertidos por la Iluvia (De Aparicio, 1931; Vignati, 1931). Sin embargo, trabajos realizados en Chile prestan especial atención a esta intervención, relacionándolas con el plano ritual y ceremonial (Massone, 1978), o con el manejo simbólico del agua en ambientes semiáridos (Castro y Gallardo, 1995-1996; Planella et al., 2017). Las canaletas que fueron registradas en el conjunto de molienda de Cerro de los Viejos son de dos tipos. Una es superficial, labrada sobre la roca soporte, pero la otra es interna uniendo un mortero aislado con una estructura combinada doble de mayor diámetro y profundidad. Esta canaleta interna pudo ser apreciada, cuando se activó durante una fuerte lluvia en uno de los trabajos de campo realizados en el Cerro (noviembre 2018). Al completarse el nivel del agua a la altura de la canaleta el agua trasvasó de un mortero al otro. Se retoman los planteos de Planella y colaboradores (2017) y Castro y Gallardo (1995-1996) referente al uso y manipulación del agua a partir de canaletas para lograr encauzar el escurrimiento del agua de lluvia y así introducir un orden simbólico al llenado de las oquedades.

Dado que el agua es un recurso crítico en la región de estudio, la dinámica cotidiana y estacional de los grupos cazadores-recolectores que habitaron este ambiente semidesértico ha estado siempre relacionada con su

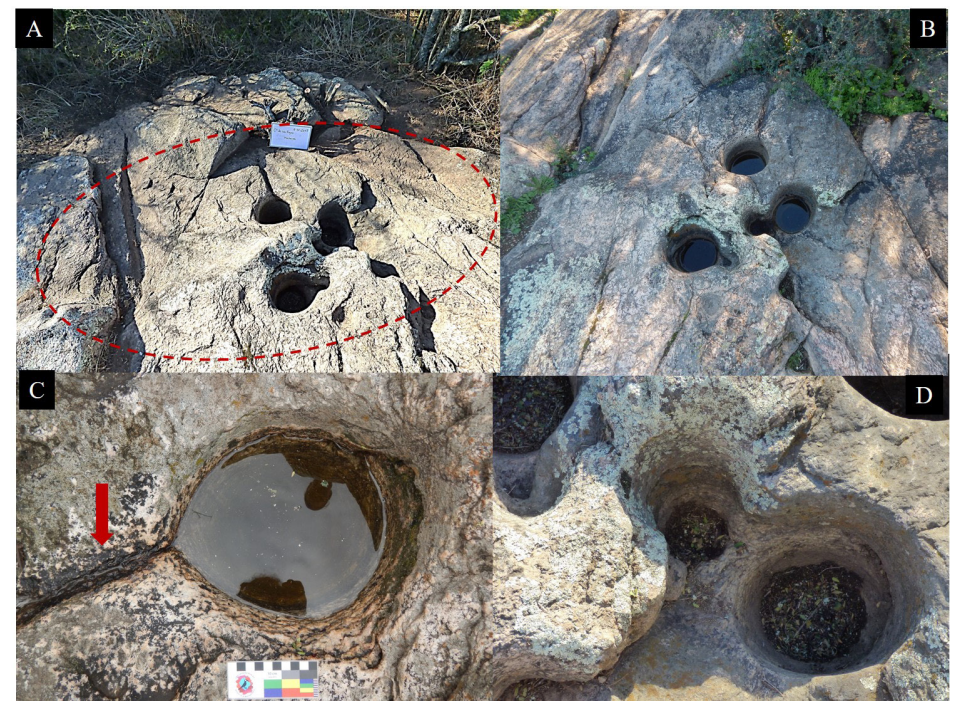

Figura 3. Referencias, A: Soporte rocoso. La línea punteada señala la superficie total donde se distribuyen los 11 morteros y los dos molinos; B: Detalle del conjunto de morteros conteniendo agua de lluvia; C: Canaleta asociada al mortero Nº10; D: Unidad compuesta de combinación doble, agrupando los morteros $\mathrm{N}^{\circ} 5$ y $\mathrm{N}^{\circ} 9$.

Figure 3. References, A: Rock support. The dotted line indicates the total area where the 11 mortars and the two grinders are distributed; B: Detail of the multiple mortar containing rainwater; $C$ : Gutter associated to mortar $N^{\circ} 10 ; D$ : Unit composed of double combination, grouping the mortars $N^{\circ} 5$ and $N^{\circ} 9$. 


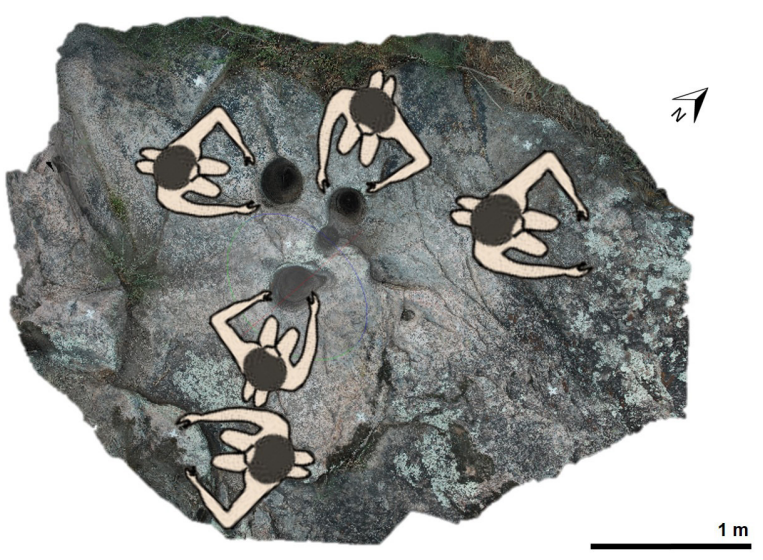

Figura 4. Reconstrucción por fotogrametría del mortero múltiple, con esquematización de la posición de los operadores (tomado y modificado de Pastor, 2010).

Figure 4. Multiple mortar photogrammetry reconstruction, with schematization of the operators position (taken and modified from Pastor, 2010).

búsqueda y aprovisionamiento, desde las exploraciones iniciales hasta tiempos históricos (Berón, 2004). Si bien no se descarta que la molienda haya sido efectivamente el modo de uso principal del conjunto de oquedades registradas en CLV4, se sostiene que quizás, a lo largo del tiempo y de generación en generación, no fue el único. Es interesante observar cómo luego de cada lluvia, el agua permanece en el interior de los morteros y se comporta como una fuente luminosa al reflejar la luz del sol que incide sobre ella, o las estrellas en el cielo nocturno. El agua ha actuado, además, durante la mayor parte de la historia humana como un espejo que permitió a las personas reflejarse y tener una imagen de sí mismas, de su entorno y de los astros (Strang, 2005).

\section{Comentarios finales}

La metodología empleada ha procurado un acercamiento al estudio del mortero múltiple de la provincia de La Pampa, que por el momento constituye el único testimonio de este tipo de estructuras. Se ha reflexionado sobre sus múltiples usos y su transgeneracionalidad. Seguramente estos bienes tuvieron múltiples significaciones para las sociedades que los crearon y utilizaron, y también para las generaciones que se sucedieron en el tiempo, incluyendo a las actuales, que ya conocen de su existencia y los resignifican.

La recurrente ocupación del Cerro durante milenios debe haber estado motivada por la disponibilidad de agua, la abundancia de recursos vegetales y animales en sus alrededores, la presencia de conjuntos artefactuales que, como en el caso de los morteros y las represas, equipaban este paisaje. El sentido de apropiación y pertenencia fue reforzado por la inhumación de los ancestros desde el menos el Holoceno Medio. El uso de ese territorio a lo largo del tiempo, por diferentes generaciones y/o poblaciones, le imprimió múltiples significados, algunos quizás compartidos, y otros que fueron re-significados por la propia dinámica social y cultural.

Ciudad Autónoma de Buenos Aires, 28 de Marzo 2019

\section{Agradecimientos}

Las investigaciones se realizaron en el marco de los proyectos ASETUR “Puesta en valor del sitio Arqueológico «Cerro de los Viejos (Cuchillo Co, La Pampa), Convenio No: 2018-23704414 Secyt, dirigido por la Dirección de Patrimonio de la Secretaría de Cultura de la provincia de La Pampa; PIP 0834 y UBACyT F-540. Agradecemos a los pobladores de Cuchillo Có y especialmente a Beto, Laura, Juan José y Susana de la familia Iturrioz por su hospitalidad y compañía en cada visita al Cerro. Agradecemos a la secretaria de la Pampa por el apoyo logístico y la infraestructura necesaria para desarrollar las tareas en el campo. También a los organizadores del Primer Congreso Argentino de Estudios Líticos en Arqueología y a la editora de la Sección Arqueología de la Revista del Museo de Antropología, por invitarnos a participar en este volumen.

\section{Bibliografía}

Adams, J. (1999). Refocusing the role of food grinding tools as correlates for subsistence strategies in the U.S Southwest. American Antiquity, 64(3), 475-498. doi:10.2307/2694147

Babot, M. P. (1999). Un estudio de artefactos de molienda. Casos del Formativo (Tesis de Grado de la Carrera de Arqueología). Facultad de Ciencias Naturales e Instituto Miguel Lillo, Universidad Nacional de Tucumán, San Miguel de Tucumán.

Babot, M. P. (2004). Tecnología y utilización de artefactos de molienda en el noroeste prehispánico (Tesis de Doctorado en Arqueología). Facultad de Ciencias Naturales e Instituto Miguel Lillo, Universidad Nacional de Tucumán, San Miguel de Tucumán.

Babot, M. P. (2007). Organización social de la práctica de molienda: casos actuales y prehispánicos del Noroeste Argentino. En A. Nielsen, M. Rivolta, V. Seldes, M. Vázquez y P. Mercolli (Eds.), Procesos sociales prehispánicos en el sur andino: la vivienda, la comunidad y el territorio (pp. 259-290). Córdoba, Argentina: Editorial Brujas.

Babot, M. P. (2009). Tradiciones, preguntas y estrategias en el abordaje arqueológico de la molienda. En R. Barberena, K. Borrazzo y L. A. Borrero (Eds.), Perspectivas actuales en arqueología argentina (pp. 147 - 188). Buenos Aires, Argentina: IMHICIHU.

Babot, M. P. (2017). Morteros de argentina. Miradas desde y hacia la arqueología de los siglos XIX y XX y prospectos para futuros estudios. En C. Belmar, L. Contreras y O. Reyes (Eds.), Piedras Tacitas. Un acercamiento integral desde la perspectiva arqueológica y patrimonial (pp. 39 - 65). Santiago de Chile: Sociedad Chilena de Arqueología y Centro de Estudios Humanos 
y Patrimoniales-Arqueólogos.

Berón, M. A. (2004). Dinámica poblacional y estrategias de subsistencia de poblaciones prehispánicas de la cuenca AtuelSalado-Chadileuvú-Curacó, provincia de La Pampa (Tesis Doctoral inédita). Facultad de Filosofía y Letras, Universidad de Buenos Aires.

Berón, M. (2019). Artefactos malacológicos. Diferentes contextos de uso en sitios arqueológicos de la provincia de La Pampa (Argentina). Comechingonia, En prensa.

Bertoldi de Pomar, H. (1975). Los silicofitolitos: sinopsis de su conocimiento. Darwiniana, 19 (24),173-206.

Bruch, C. (1911). Exploraciones arqueológicas en las provincias de Tucumán y Catamarca. Biblioteca Centenaria, 5, 25 y sig.

Cabrera, A. (1971). Fitogeografía de la República Argentina. Boletín de la Sociedad Argentina de Botánica, 14, 1-42.

Carrera Aizpitarte, M., Luna, L., Aranda, C. y Berón, M. (2013). Médano La Enriqueta: un lugar de entierro cazadores tardíos sobre el río Colorado (Dpto. Caleu Caleu, provincia de La Pampa). Revista del Museo de La Plata. Sección Antropología, 13 (87), 167-184.

Castro, V. y Gallardo, F. (1995-1996). El poder de los gentiles. Arte rupestre en el río Salado. Revista Chilena de Antropología, 13, 78-98. doi: 10.5354/0719-1472.2011.17521

De Aparicio, F. (1925). Investigaciones arqueológicas en la región serrana de la provincia de Córdoba. (Breve noticia preliminar). Anales de la Sociedad Argentina de Estudios Geográficos "Gaea", 1, 120-144.

De Aparicio, F. (1931). La vivienda natural en la región serrana de Córdoba. Buenos Aires: Publicaciones del Museo Antropológico y Etnográfico de la Facultad de Filosofía y Letras, Serie A (1).

Flannery, K. V. (1986). Ground-stone artifacts. En K. V. Flannery (Ed.), Guilá Naquitz: Archaic Foraging and Early Agriculture in Oaxaca, Mexico (pp. 147-156). Nueva York: Academic Press.

Giovannetti, M. (2009). Los morteros múltiples en el Noroeste argentino. Un enfoque integral. En A. Austral y M. Tamagnini (Comp.), Problemáticas de la arqueología contemporánea, III (pp. 773-782). Rio Cuarto, Argentina: Universidad Nacional de Rio Cuarto.

Giovannetti, M. (2013). Propuesta para la recolección de microvestigios arqueobotánicos en morteros fijos. Comechingonia. Revista de Arqueología, (17), 189-199.

Goñi, R.A. (1986-1987). Arqueología de sitios tardíos en el valle del río Malleo, Pcia. Del Neuquén. Relaciones de la Sociedad Argentina de Antropología, XVII (1), 37-66.

Gosden, C. y Marshall, Y. (1999). The Cultural Biography of Objects. World Archaeology, 31 (2), 169-178 doi: 10.1080/00438243.1999.9980439

Gradín, C., Vayá, C., Quintana, M., Nami, H., Salvino, A., Berón, M. y Aguerre, A. (1984). Investigaciones Arqueológicas en Casa de Piedra. Pcia. de La Pampa. La Pampa: Dirección General de Cultura y Ente Ejecutivo Casa de Piedra.
Hayden, B. (1987). Past to present uses of stone tools and their effects on assemblage characteristics in the Maya Highlands. En B. Hayden (Ed.), Lithic Studies Among the Contemporary Highland Maya (pp 160-234). Tucson: University of Arizona Press.

Hermosilla Osorio, N. (2017). Las piedras tacitas como enclaves culturales. En C. Belmar, L. Contreras y O. Reyes (Ed.), Piedras Tacitas. Un acercamiento integral desde la perspectiva arqueológica y patrimonial (pp. 195 - 201). Santiago de Chile: Sociedad Chilena de Arqueología y Centro de Estudios Humanos y Patrimoniales-Arqueólogos.

Hermosilla, N. y Ramírez, J. (1985). Las Cenizas: Evidencias de Ritualismo en torno a las piedras tacitas. Actas del IX Congreso Nacional de Arqueología Chilena (pp. 307-320). Museo Arqueológico de La Serena, La Serena.

ICSN. (2011). The International Code for Starch Nomenclature. www.fossilfarm.org/ICSN/Code.html (último acceso 2011).

Instituto Nacional de Tecnología Agropecuaria (INTA). (1980). Inventario integrado de los recursos naturales de la provincia de La Pampa. Clima, geomorfología, suelo y vegetación. Universidad Nacional de La Pampa, Buenos Aires.

Jackson, S. D. (2004). Los implementos de molienda en un campamento estacional del Holoceno medio: implicancias funcionales y contextuales. Chungara Revista de Antropología Chilena, 36 (1), 95-103. doi:10.4067/S071773562004000300012.

Keane, W. (2005). Signs are not the garb of meaning. On the social analysis of material things. En D. Miller (Ed.), Materiality (pp. 182-205). Durham: Duke University Press. doi: 10.1215/9780822386711-008

Ledesma, R. (1961). Maquijata. Santiago del Estero, Argentina: Editorial de la UNT Facultad de Filosofía y Letras, Instituto de Lingüística, Folklore y Antropología.

Madella, M., Alexandre, A. y Ball, T. (2005). International Code for Phytolith Nomenclature 1.0. Annals of Botany, 96(2), 253260. doi: $10.1093 /$ aob/mci172

Madrid, P. (1991). Infraestructura indígena para el mantenimiento y traslado de ganado introducido: el caso del Sistema Serrano de Pillahuinco, pcia. de Bs. As. Boletín del Centro, 3, 65-71.

Mandrini, R. (1984). Los araucanos de las pampas en el siglo XIX. Historia Testimonial Argentina 22. Buenos Aires, Argentina: Centro Editor de América Latina.

Mandrini, R. (1991). Procesos de especialización regional en la economía indígena pampeana (s. XVIII y XIX): el caso del suroeste bonaerense. Boletín Americanista 41, 113-136.

Mansur-Franchomme, M., Orquera, L. y Piana, E. (1987-88). El alisamiento de la piedra entre cazadores-recolectores: el caso de Tierra del Fuego. Runa 17-18, 111-205.

Massone, M. (1978). Cerro Blanco. Antropología de un asentamiento humano. (Tesis para optar al grado de Licenciado en Antropología Social). Departamento de Ciencias Antropológicas y Arqueológicas, Universidad de Chile, Santiago. 
Miller, D. (2005). Materiality: an introduction. En D. Miller (Ed.), Materiality (pp. 1-50). Durham: Duke University Press.

Moreyra, C. E. (2011). Pensar los objetos. Problemas y fuentes para el estudio de la cultura material en la época colonial. Anuario de Arqueología, 3 (3), 121-141.

Musaubach, M. G. (2014). Estudios Arqueobotánicos en sociedades cazadoras-recolectoras de ambientes semiáridos Análisis de microrestos vegetales en contextos arqueológicos de Pampa Occidental (Argentina). (Tesis de Doctorado inédita). Facultad de Filosofía y Letras, Universidad de Buenos Aires.

Musaubach, M. G. (2017). Informe interno de análisis arqueobotánicos de Cerro de los Viejos. MS.

Nardi, R. y Chertudi, S. (1969). Instrumentos arcaicos para majar y moler en San Juan (Argentina). Revista de Etnografía, 13 (26), 387-418.

Núñez, L. y Santoro, C. (1990). Primeros poblamientos en el cono Sur de América (XII-IX milenio A.P.). Revista de Arqueología Americana 1, 91-139.

Palermo, M. (1986). Reflexiones sobre el llamado "complejo ecuestre" en la Argentina. Runa XVI, 157-178.

Palermo, M. (1991). La compleja integración hispano-indígena del sur Argentino-Chileno durante el período colonial. América Indígena, LI (1), 153-192.

Pastor, S. (2010). Aproximación inicial a la arqueología del norte de la sierra de Guasapampa y cordón de Serrezuela (Córdoba, Argentina). Arqueología, 16, 151-174.

Pastor, S. (2015). Acerca de la constitución de agentes sociales, objetos y paisajes. Una mirada desde las infraestructuras de molienda (Sierras de Córdoba, Argentina). En J. Salazar (Comp.), Condiciones de posibilidad de la reproducción social en sociedades prehispánicas y coloniales tempranas en las Sierras Pampeanas (República Argentina) (pp. 302-341). Córdoba, Argentina: Centro de Estudios Históricos Prof. Carlos S.A. Segreti.

Pedrotta, V. (2016). Estrategias indígenas de captura y manejo del ganado cimarrón en las Sierras Septentrionales bonaerenses: las construcciones de piedra Cerro Guacho I y Cerro Guacho II. Arqueología, 22(2), 269-289. doi:10.34096/arqueologia. t22. n2.3206.

Petrucci, N. S. (2016). Organización espacial de la molienda en el sitio arqueológico Rincón Chico 1 (Catamarca, Argentina). Revista del Museo de Antropología, 9 (2), 7-16. doi:10.31048/1852.4826.v9.n2.15889.

Piana, E. (1981). Toponimia y Arqueología del siglo XIX. Buenos Aires, Argentina: Eudeba.

Pino, M., Troncoso, A., Belmar, C. y Pascual, D. (2018). Bedrock mortars in the semiarid north of Chile $\left(30^{\circ} \mathrm{s}\right.$.): time, space, and social processes among late Holocene hunter-gatherers. Latin American Antiquity, 1-20. doi:10.1017/laq.2018.52.

Planella Ortiz, M. T., Santander Horta, G. y McRostie Bustamante, V. (2017). Aportes a la discusión sobre piedras tacitas en Chile Central. Intersecciones en Antropología, 18, 05-17.

Schäbitz, F. (1994). Holocene climatic variations in northern Patagonia, Argentina. Palaeogeography, paleoclimatology, palaeoecology, 109, 287-294.

Schlanger, S. (1991). On manos, metates, and the history of site occupations. American Antiquity, 54, 460-474.

Strang, V. (2005). Common senses. Water, Sensory Experience and the Generation of Meaning. Journal of Material Culture, 10(1), 92-120. doi:10.1177/1359183505050096

Tapia, A. y Charlin, J. (2004). Actividades de molienda y pulido en las tolderías ranquelinas del caldenar pampeano. En G. Martínez, M. Gutiérrez, R. Curtoni, M. Berón y P. Madrid (Eds.), Aproximaciones Contemporáneas a la Arqueología Pampeana. Perspectivas teóricas, metodológicas, analíticas y casos de estudio (pp. 363385). Olavarría: Facultad de Ciencias Sociales (UNCPBA).

Tickyj, H., Dimieri, L. V., Llambias, E. J. y Sato, A. M. (1997). Cerro de Los Viejos ( $38^{\circ} 28^{\prime} \mathrm{S}-64^{\circ} 26^{\prime} 0$ ): cizallamiento dúctil en el sudeste de La Pampa. Revista de la Asociaci6n Geológica Argentina, 52 (3), 311-321.

Tilley, C. (1999). The metaphorical transformations of wala canoes. En V. Buchli (Ed.), The material culture reader (pp. 102-132). Oxford: Berg.

Vecchi, R. y González, M. I. (2018). Artefactos formatizados por picado, abrasión, pulido y modificados por uso en la depresión del Río Salado, Provincia de Buenos Aires, Argentina. Revista del Museo de Antropología, 11 (2), 85-100.

Vignati, M. (1931). ¿Morteros o represas? Nueva interpretación de las agrupaciones de "morteros". Notas Preliminares del Museo de La Plata, 1, 45-61.

Wright, K., (1994). Ground-stone tools and hunter gatherer subsistence in Southwest Asia: Implications for the transition to farming. American Antiquity, 59 (2), 238-263. 